Review began 08/12/2021 Review ended 08/19/2021 Published 09/05/2021

๑) Copyright 2021

Nishimura et al. This is an open access article distributed under the terms of the Creative Commons Attribution License CCBY 4.0., which permits unrestricted use, distribution, and reproduction in any medium, provided the original author and source are credited.

\section{Variation of Tumor Volume During Moderate Hypo-Fractionated Stereotactic Body Radiation Therapy for Lung Cancer} Keiichiro Nishimura ${ }^{1}$, Shogo Hatanaka ${ }^{1}$, Nobuko Utsumi ${ }^{2,3}$, Takafumi Yamano ${ }^{1}$, Munefumi Shimbo ${ }^{1}$,
Takeo Takahashi $^{1}$

1. Department of Radiation Oncology, Saitama Medical Center, Saitama Medical University, Kawagoe, JPN 2. Department of Radiation Therapy, JCHO Tokyo Shinjuku Medical Center, Tokyo, JPN 3. Department of Radiation Oncology, Saitama Medical Center, Saitama Medical University, Saitama, JPN

Corresponding author: Shogo Hatanaka, hatasho@saitama-med.ac.jp

\section{Abstract}

\section{Aim}

To investigate the variation of tumor volume during moderate hypo-fractionated stereotactic body radiation therapy (SBRT).

\section{Patients and Methods}

Twenty patients, who received SBRT at our institution, were included in the analysis. A prescribed dose was 56 Gy at iso-center in seven fractions. Tumor volumes before and during SBRT were calculated. In order to investigate factors affecting the variation of tumor volume in RT 2 (after first irradiation) and RT 7 (after last irradiation), various parameters were verified by the Mann-Whitney $U$ test.

\section{Results}

With regard to the low maximum standardized uptake values (SUVmax) group, transient increase of tumor volume was found in RT 2, and tumor volume reduction was hardly found in RT 7. With regard to the high SUVmax group, a transient increase was not found, and a definite reduction was found in the treatment course.

\section{Conclusion}

Accurate prediction of tumor volume variation is required for more accurate treatment, such as adaptive radiation therapy.

Categories: Radiation Oncology, Oncology

Keywords: stereotactic body radiation therapy (sbrt), moderate hypo-fractionated sbrt, lung cancer, variation of tumor volume, maximum standardized uptake values

\section{Introduction}

Stereotactic body radiation therapy (SBRT) is a treatment technique for irradiation to localized cancer in a trunk of a body accurately. With this technique, total doses of radiation are divided into large doses, and radiation treatment is given over a shorter period compared to conventional radiation therapy. At present, the SBRT technique has been used widely for the treatment of stage I non-small cell lung cancer [1]. In Japan, SBRT with 48 Gy in four fractions is implemented at many institutions according to Japan Clinical Oncology Group 0403 protocol [2, 3]. Ohnishi et al. reported that the 5-years survival rate was $72 \%$, and the local control rate was 87-97区 by using SBRT for stage I non-small cell lung cancer in a multi-institutional study under the condition that biological effective doses (BED) were $\geqslant 100 \mathrm{~Gy}(\alpha / \beta=10 \mathrm{~Gy})$ [4]. Similarly, by using SBRT with 48 Gy in four fractions, Nagata et al. reported that the 3-years survival rate was $83 \%$ [5]. However, both reports investigated only for peripheral lung cancer, and central lung cancer was not included. In a previous report, grade 5 adverse events were found after SBRT in patients of central lung cancer with 48 Gy in 4 fractions [6]. Therefore, regarding central lung cancer, moderate hypo-fractionated SBRT (e.g., $56 \mathrm{~Gy}$ in seven fractions) might be useful for the reduction of late effect in normal tissues.

In radiation therapy, the accurate decision of gross tumor volume (GTV) and target volume is important; thus, it is required to consider the variation of tumor volume during the course of treatment. Recently, even for SBRT with a short period of treatment, variation of tumor volume during the course of treatment has been investigated in several reports [7-9]. Regarding SBRT with 48-52 Gy in four fractions, Tatekawa et al. reported $\geqslant 10 \%$ increase in tumor volume was detected in 16 of 50 cases. For that reason, considering the tumor volume is important in order to perform a more accurate treatment, such as adaptive radiation therapy [7]. However, a mechanism of this transient increase during SBRT is not clear. Additionally, no 


\section{Cureus}

report has verified variation of tumor volume during moderate hypo-fractionated SBRT. Therefore, we investigated the variation of tumor volume during moderate hypo-fractionated SBRT in this study.

\section{Materials And Methods}

Table 1 shows patient information. From April 2015 to December 2016, 20 patients (21 plans), who received SBRT at our institution, were included in the analysis. All procedures were approved by the Ethical Committee of our institution.

\begin{tabular}{|c|c|c|}
\hline \multirow{2}{*}{ Gender } & Male & Female \\
\hline & 14 & 6 \\
\hline \multirow{2}{*}{ Age } & rage & mean \\
\hline & $67-88$ & 76 \\
\hline \multirow{2}{*}{ Operation history of lung } & yes & no \\
\hline & 6 & 15 \\
\hline Pathology & \multicolumn{2}{|l|}{$\square$} \\
\hline adenocarcinoma & \multicolumn{2}{|l|}{10} \\
\hline squamous cell carcinoma & \multicolumn{2}{|l|}{1} \\
\hline non-small cell carcinoma & \multicolumn{2}{|l|}{1} \\
\hline unknown & \multicolumn{2}{|l|}{5} \\
\hline metastases* & \multicolumn{2}{|l|}{4} \\
\hline \multicolumn{3}{|c|}{ * primary lesion (rectum 1 , esophagus 1 , renal cell 1 , ureteral 1 ) } \\
\hline \multirow{8}{*}{ Tumor site } & distal side & central side \\
\hline & 20 & 1 \\
\hline & left lobe & right lobe \\
\hline & 9 & 12 \\
\hline & superior side & inferior side \\
\hline & 15 & 6 \\
\hline & anterior side & posterior side \\
\hline & 12 & 9 \\
\hline \multirow{2}{*}{ Tumor size } & range & mean \\
\hline & $7-39 \mathrm{~mm}$ & $22.8 \mathrm{~mm}$ \\
\hline \multirow{2}{*}{ Destruction of alveolar wall around the tumor } & yes & no \\
\hline & 9 & 12 \\
\hline
\end{tabular}

\section{TABLE 1: Patient information}

\section{2-1. Treatment planning}

SFORM ESN-1800 (Engineering system) was used for a patient fixture. The breathing of the patient was suppressed by chest compression. computed tomography (CT) images for dose calculation were acquired by GE Optima CT580 (GE Medical Systems, Chicago, Illinois) with a slow scan method in four seconds per rotation under the condition of free breathing. The slice thickness was $1.25 \mathrm{~mm}$, tube voltage was $120 \mathrm{kV}$, and automatic exposure control was used to regulate tube current ( $36.6 \mathrm{~mA}$ to $500 \mathrm{~mA}$ ). XiO (Elekta, Stockholm, Sweden) was used as the radiation treatment planning system (RTPS). The linear accelerator Clinac21EX (Varian Medical Systems, Helsinki, Finland) with a 6 MV X-ray was used as a radiation source. Created plans contained non-coplanar 8-10 fields. The GTV and internal target volume (ITV) were 


\section{Cureus}

determined based on CT images. The planning target volume (PTV) was defined by the ITV with a seven mm margin. The radiation fields were optimized to fit the PTV with a multi-leaf collimator (MLC) margin of five $\mathrm{mm}$. A prescribed dose was $56 \mathrm{~Gy}$ at iso-center in seven fractions (three fractions per week). Figure 1 shows a summary of the treatment course.

\begin{tabular}{|c|c|c|c|c|c|c|c|c|c|}
\hline Days & \multicolumn{2}{|c|}{-5} & 1 & 3 & 5 & 8 & 10 & 12 & 15 \\
\hline $\mathrm{CT}$ image set number & (1) & (2) & (3) & (4) & (5) & (6) & (7) & (8) & (9) \\
\hline Planning / Treatment & \multicolumn{2}{|c|}{$\begin{array}{l}\text { Treatment } \\
\text { planning }\end{array}$} & \multicolumn{7}{|c|}{ SBRT treatment } \\
\hline Symbol in this paper & & & RT 1 & RT 2 & RT 3 & RT 4 & RT 5 & RT 6 & RT 7 \\
\hline eference / Meas & $\begin{array}{l}\text { Refe } \\
\text { (me }\end{array}$ & ence i & mage & \multicolumn{6}{|c|}{$\begin{array}{l}\text { Measurement of change of } \\
\text { tumor volume and tumor center } \\
\text { position }\end{array}$} \\
\hline
\end{tabular}

FIGURE 1: Schedule of Stereotactic Body Radiotherapy

\section{2-2. Variation of tumor volume during SBRT}

An automatic contouring function installed in Velocity 3.2.1 (Varian Medical Systems, Helsinki, Finland) was used for acquiring tumor volume in this study. A threshold value of a CT value was - 250 Hounsfield units (HU) [7]. In addition, one experienced radiation oncologist excluded blood vessels and chest walls manually. Figure 2 shows an example of a tumor contour. Ratios of reference tumor volume (TVR) and tumor volume during SBRT (TV2-TV7) were calculated (TVX/TVR). An average value of tumor volume which was acquired by three CT images (1st planning CT, 2nd planning CT, and RT 1 CT images) was used as TVR (see Figure 1).

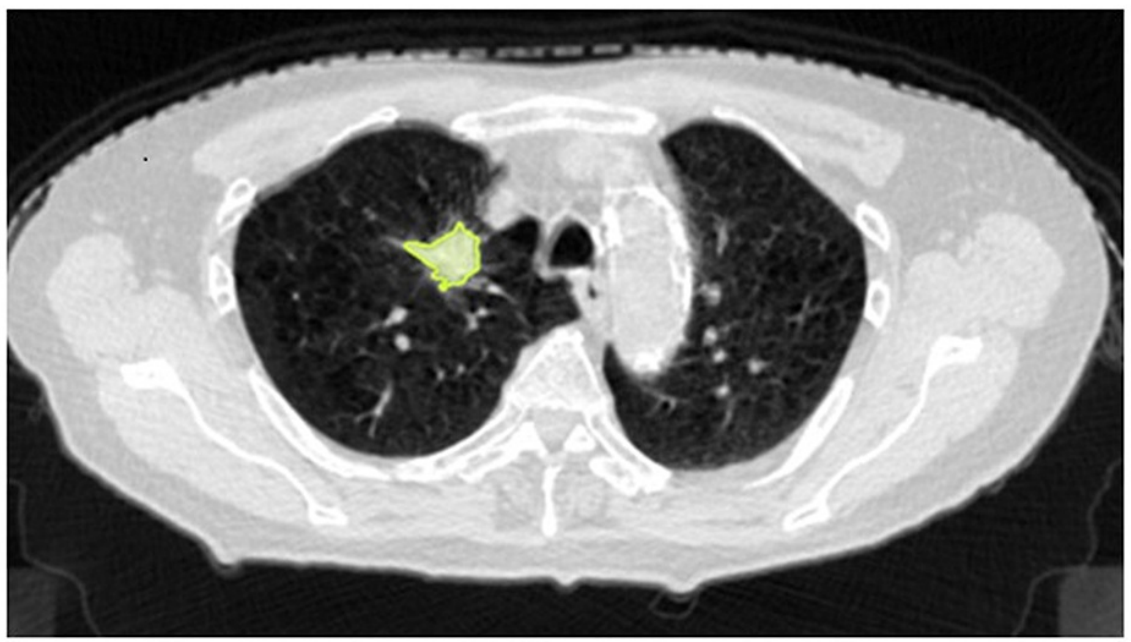

\section{FIGURE 2: Contour extraction for SBRT}

An automatic contouring function installed in Velocity software was used for acquiring tumor volume in this study. A threshold value of a CT value was $-250 \mathrm{HU}$. In addition, one experienced radiation oncologist excluded blood vessels and chest walls manually.

\section{2-3. Statistical analysis}

In order to investigate factors affecting the variation of tumor volume in RT 2 (after first irradiation) and RT 
7 (after last irradiation), the following parameters were verified by the Mann-Whitney U test. Gender, Age (< 70 or $\geqslant 70$, and < average:76 or $\geqslant 76$ ), Presence of lung surgery anamnesis, tumor volume before SBRT (<average $\mathbf{X} 4.4 \mathrm{~cm}^{3}$ or $\geqslant 4.4 \mathrm{~cm}^{3}$ ), lung volume (< average $\mathbf{X} 2875 \mathrm{~cm}^{3}$ or $\geqslant 2875 \mathrm{~cm}^{3}$ ), Presence of alveolar wall destruction near tumors, tumor locations (peripheral or central, left or right lobe, head or foot side, and ventral or dorsal side), and maximum standardized uptake values (SUVmax) acquired by 18F-FDGPositron Emission Tomography (PET) images. FDG-PET / CT is widely used and the most reliable modality to evaluate the tumor metabolic activity using the glucose metabolism independent of morphological change. Although various parameters in FDG-PET/CT have been reported, SUVmax is the most common and reliable parameter to evaluate tumor metabolic activity. Since this study is a retrospective study, the constantly measured and reliable SUVmax was used as a biomarker in this study. The tumor locations of head-foot sides were distinguished by halfway lines between lung apexes and bottoms as boundaries. The tumor locations of ventral-dorsal sides were distinguished by vertebral leading edges as boundaries.

\section{Results}

Figure 3 shows ratios of reference tumor volume and tumor volume during SBRT and results of paired t-test. A definite increase was found in TV2; on the other hand, a definite decrease was found in TV7. Results of statistical analysis for variation of tumor volume (TV2/TVR and TV7/TVR) were shown in Table 2. Regarding gender, age, presence of lung surgery anamnesis, tumor volume before SBRT, lung volume, presence of alveolar wall destruction, and tumor location, we did not find a significant association between these and the variation of tumor volume. On the other hand, a TV2/TVR mean value of SUVmax in the < average group was larger than a TV2/TVR mean value of SUVmax in the $\geqslant$ average group $(p<0.02)$ as showed in Figure 4 . Similarly, a TV7/TVR mean value of SUVmax in the < average group was larger than a TV7/TVR mean value of SUVmax in the $\geqslant$ average group $(p<0.05$ ), as showed in Figure 5. Figure 6 shows a comparison of variation in TVX/TVR during SBRT between values of SUVmax in the ₹ average group and values of SUVmax in the $\geqslant$ average group. With regard to the values of SUVmax in the < average group, a transient increase of tumor volume was found in RT 2, and tumor reduction was hardly found in the latter half of the treatment course. With regard to the values of SUVmax in the ₹ average group, a transient increase of tumor volume was not found, and definite tumor reduction was found in the latter half of the treatment course.

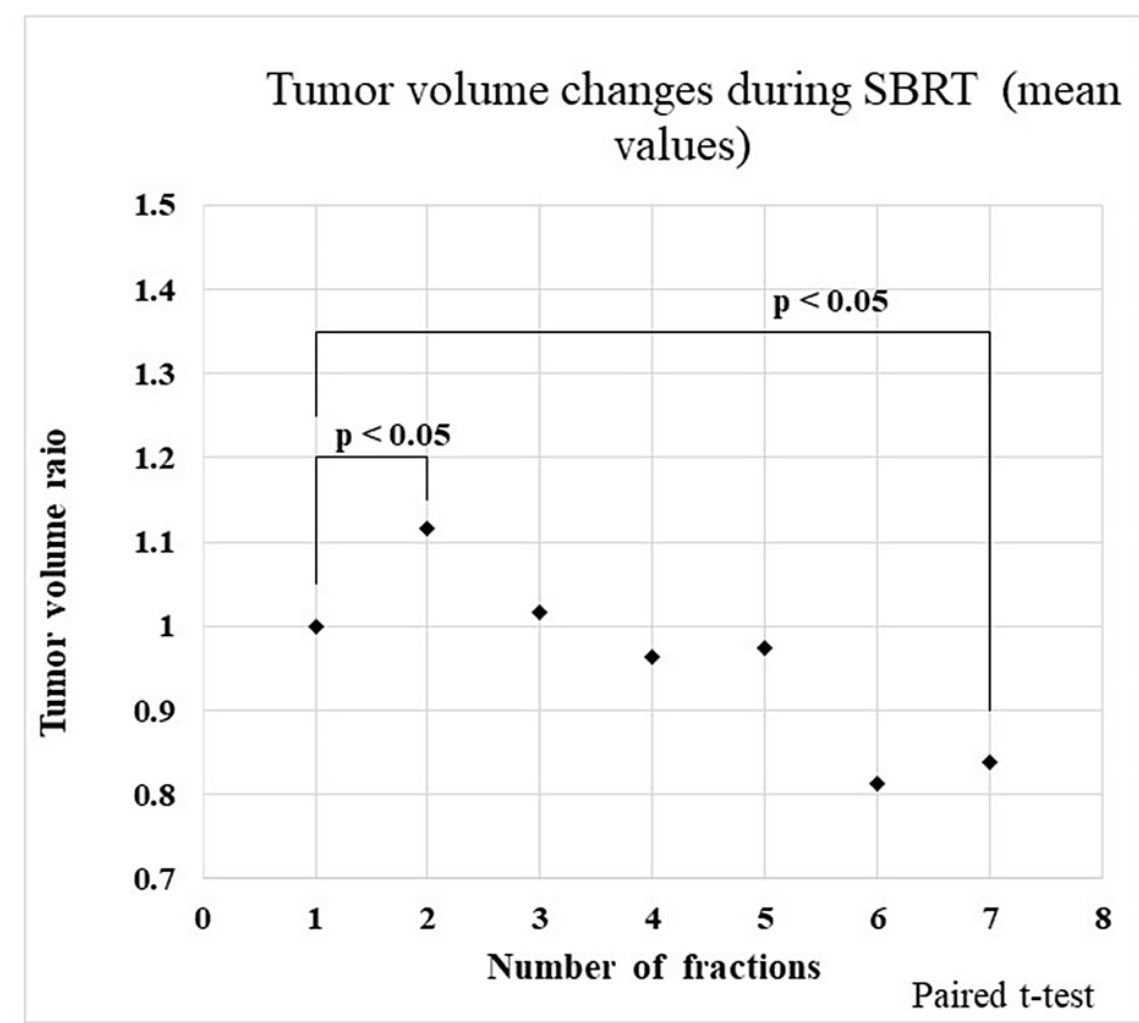

FIGURE 3: Tumor volume changes during SBRT (mean values)

A definite increase was found in TV2, on the other hand, a definite decrease was found in TV7.

SBRT - stereotactic body radiotherapy 


\section{Cureus}

Comparison of tumor volume ratio between

SUVmax $<9.38$ and $\geqq 9.38$ at RT 2

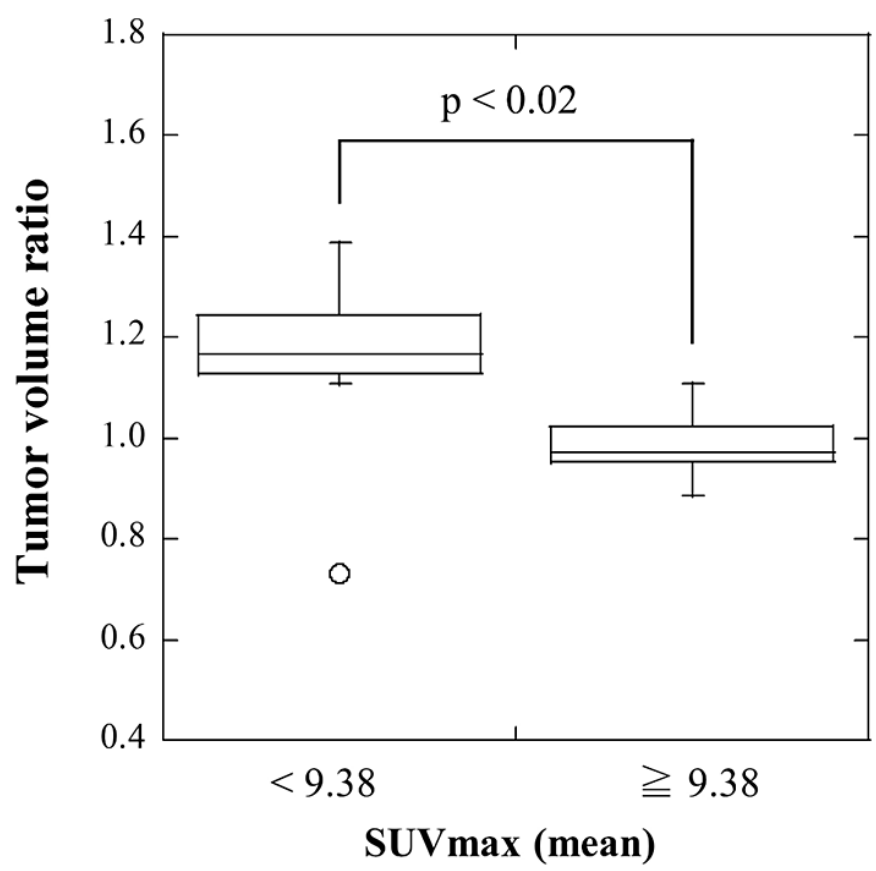

Mann - Whitney $U$ Test

FIGURE 4: Comparison of tumor volume ratio between SUVmax $<9.38$ and $\geq 9.38$ at RT2

A TV2/TVR mean value of SUVmax in the < average group was larger than a TV2/TVR mean value of SUVmax in the $\geq$ average group $(p<0.02)$.

SUVmax - maximum standardized uptake values 


\section{Cureus}

Comparison of tumor volume ratio between

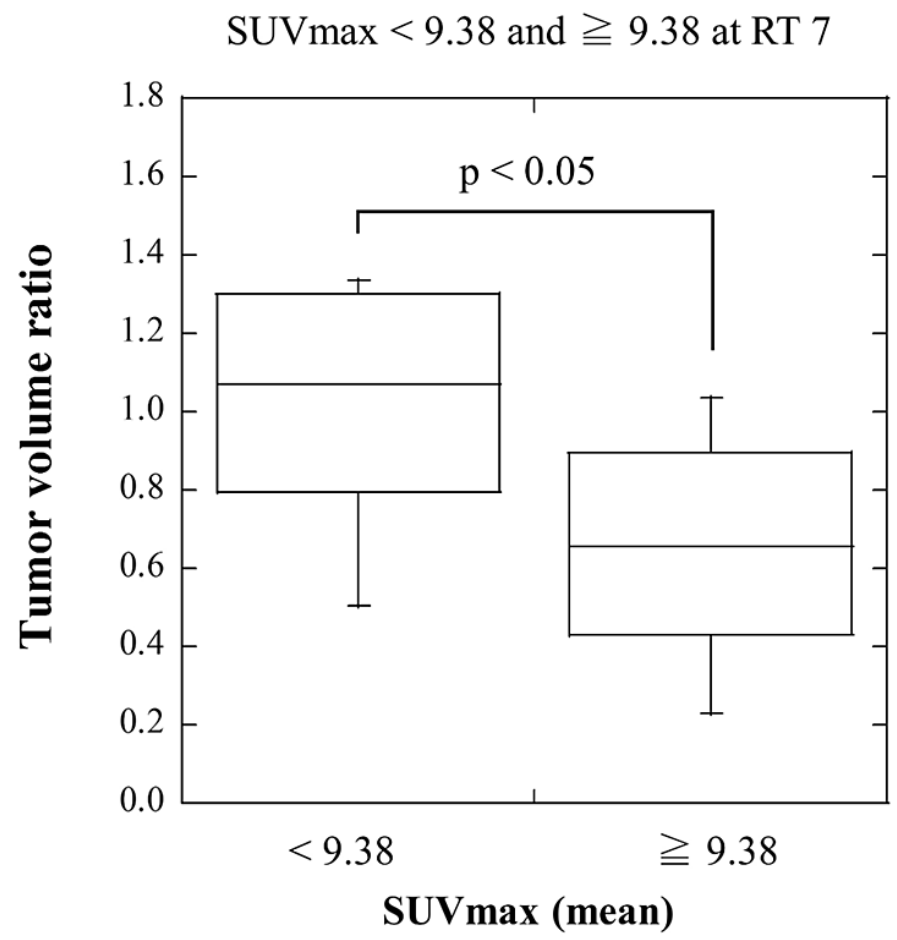

Mann - Whitney $U$ Test

FIGURE 5: Comparison of tumor volume ratio between SUVmax $<9.38$ and $\geq 9.38$ at RT7.

A TV7/TVR mean value of SUVmax in the < average group was larger than a TV7/TVR mean value of SUVmax in the $\geq$ average group $(p<0.05)$.

SUVmax - maximum standardized uptake values 


\section{Cureus}

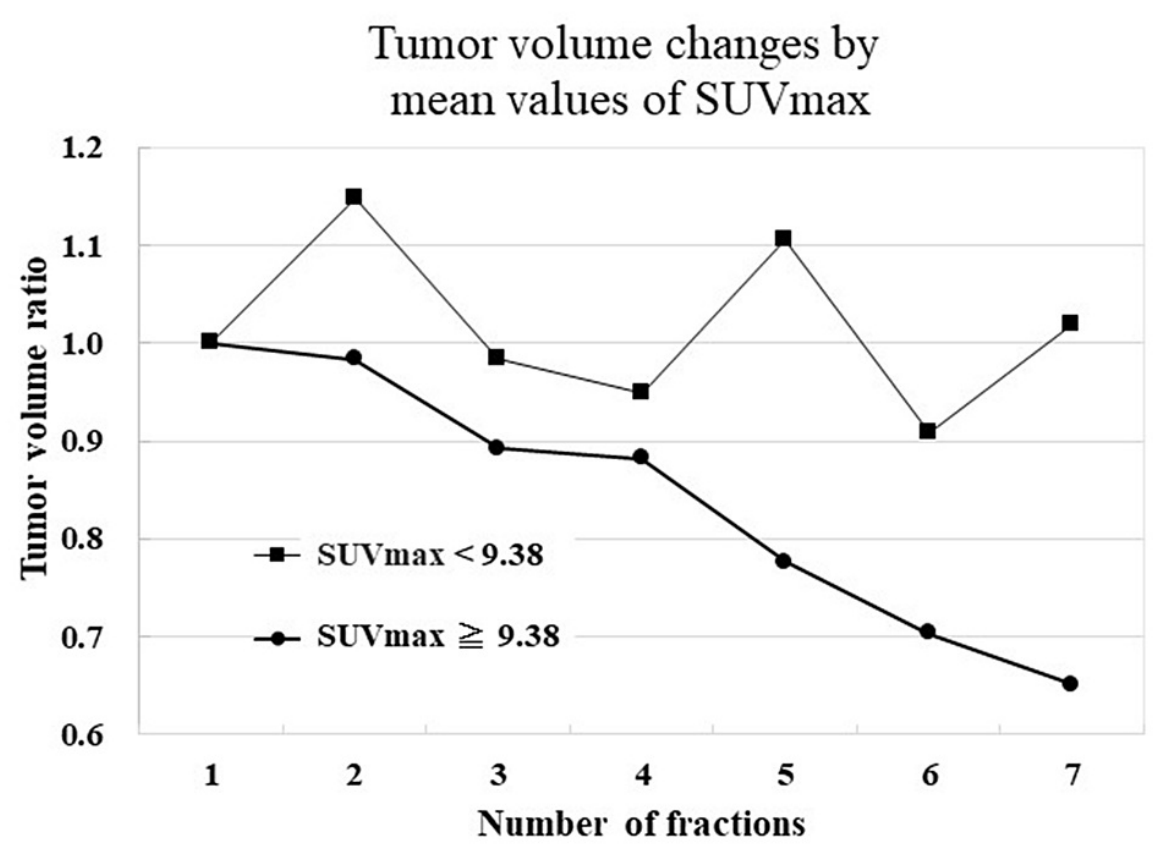

FIGURE 6: Tumor volume changes by mean values of SUVmax

With regard to the values of SUVmax in the < average group, a transient increase of tumor volume was found in RT 2, and tumor reduction was hardly found in the latter half of the treatment course. With regard to the values of SUVmax in the $\geq$ average group, a transient increase of tumor volume was not found, and definite tumor reduction was found in the latter half of the treatment course.

SUVmax - maximum standardized uptake values

\begin{tabular}{|c|c|c|c|c|}
\hline$\square$ & \multicolumn{2}{|l|}{ Group } & \multirow{2}{*}{$\begin{array}{l}\mathrm{TV}_{2} / \mathrm{TV}_{\mathrm{R}} \\
\text { N.S. }\end{array}$} & \multirow{2}{*}{$\begin{array}{l}\mathrm{TV}_{7} / \mathrm{TV}_{\mathrm{R}} \\
\text { N.S. }\end{array}$} \\
\hline Gender & Male & Female & & \\
\hline Operation history of lung & yes & no & N.S. & N.S. \\
\hline Tumor volume & $\square 4.4 \square^{3}$ & $\geqq 4.4 \square^{3}$ & N.S. & N.S. \\
\hline Lung volume & $\square 2875 \square^{3}$ & $\geqq 2875 \square^{3}$ & N.S. & N.S. \\
\hline Destruction of alveolar wall around the tumor & yes & no & N.S. & N.S. \\
\hline \multirow{4}{*}{ Tumor site } & distal site & central site & N.S. & N.S. \\
\hline & left lobe & right lobe & N.S. & N.S. \\
\hline & superior side & inferior side & N.S. & N.S. \\
\hline & anterior side & posterior side & N.S. & N.S. \\
\hline SUVmax & $\square 9.38$ & $\geqq 9.38$ & $\mathrm{p} \square 0.02$ & $\mathrm{p} \square 0.05$ \\
\hline
\end{tabular}

TABLE 2: Results of statistical analysis for variation of tumor volume (TV2/TVR and TV7/TVR)

N.S. - not significant

\section{Discussion}

Regarding chemo-radiotherapy with standard fractionated irradiation, which requires six weeks for 
treatment, it has been reported that reduction of tumor volume was found in 40-50\% cases with non-small cell lung cancer $[10,11]$ and was found in $65 \%$ cases with small cell lung cancer [10]. Variation of tumor volume during the treatment course had not been investigated until a while ago because the treatment period of SBRT was very short (about one week). However, recently Tatekawa K et al. [7] and Gunter T et al. [8] reported that transient increase of tumor volume during treatment course in SBRT with 4-5 fractions was found. Bhatt et al. had reported that a decrease of tumor volume during SBRT with five fractions was found [9]. In regard to this study, with moderate hypo-fractionated SBRT, which requires 2.5 weeks for treatment, a definite transient increase of tumor volume was found in RT 2. It is unlikely that the tumor growth is found during the short treatment course of SBRT using a large dose per fraction compared with conventional radiotherapy. Because it is thought that the time when the tumor increases transiently in RT2 of SBRT does not coincide with the time when repopulation of the tumor occurs. Therefore, it was considered that the transient increase of the tumor was not due to the growth of the tumor but due to the effect of irradiation on the interstitial component of the tumor, such as edema caused by large irradiation dose per fraction [7]. On the other hand, a definite reduction of tumor volume was found in RT 7. Regarding gender, age, presence of lung surgery anamnesis, tumor volume before SBRT, lung volume, presence of alveolar wall destruction, and tumor locations, we found no significant association between these and variation of tumor volume. Verifying the relationship between SUVmax value and variation of tumor volume, trends were different between a high SUVmax value group and a low SUVmax value group. A high proliferation rate for cells and a high growth rate for tissues result in increased radiosensitive. Investigation of the relationship between the expression of biomarkers related to tumor proliferation and radio-sensitivity had been implemented [12]. However, for radiotherapy of small lung cancer, the use of biomarkers obtained by tumor tissues was difficult. Therefore, SUVmax values obtained by 18F-FDG-PET/CT images were used for a biomarker in this study. It has been reported that 18F-FDG-PET/CT has high sensitivity (96.8\%) and moderate specificity (77.8\%) for the diagnosis of lung cancer [13]. SUVmax values and tumor proliferation were correlated [14, 15]. In a high SUVmax value group, tumor reduction was found in an early stage of the treatment course because tumor proliferation rates and radio-sensitivity might be high. On the other hand, in a low SUVmax value group, a transient increase of tumors was found in an early stage of the treatment course because of the influence of interstitial change such as edema caused by large radiation doses [7].

As a limitation, a slow-scan technique was used for the acquisition of CT images in this study. Regarding the accurate acquisition of moving tumor shapes, the slow scan is inferior to high-speed scan techniques such as $4 \mathrm{D}-\mathrm{CT}[16,17]$. However, a slow scan can acquire tumor central position and a moving range accurately [16, 17]. Therefore, we expected that a slow scan CT could be used for the evaluation of relative tumor volume variation.

From now on, an increasing ratio of elderly patients with lung cancer is predicted. Sandhu AP et al. had reported that the 2-years disease-free survival rate was $77 \%$, and the 2 -years overall survival rate was $74 \%$ for elderly patients from 80 to 90 years old with stage I non-small cell lung cancer by using SBRT [18]. Additionally, they had reported that SBRT was a useful choice for the treatment of elderly patients with early non-small cell lung cancer [18]. Therefore, for patients who cannot undergo surgery with stage I-II nonsmall cell lung cancer, metastatic lung cancer, and liver cancer, SBRT might be a better choice for treatment $[18,19]$. The utility and safety of SBRT with 4 fractions for peripheral lung cancer are established; however, an increase of adverse events is concerned for patients with central lung cancer. Haseltine JM et al. had investigated adverse events after SBRT with $48 \mathrm{~Gy}$ in four fractions - $50 \mathrm{~Gy}$ in five fractions for central lung cancer [20]. According to this report, when the distance from a bronchial tube to a tumor was $<1 \mathrm{~cm}$, the frequency of adverse events of $\geqslant$ grade 3 was definitely increased [20]. On the other hand, in SBRT with an increased number of fractions, a decrease of serious adverse events was found [21, 22]. In the SBRT phase I trial (JROSG 10-1) for stage IA central non-small cell lung cancer, it had been reported that SBRT with 60 Gy in eight fractions was appropriate for stage IA central lung cancer from a perspective of adverse events [23]. Shibamoto et al. had reported that SBRT with $60 \mathrm{~Gy} /$ eight fractions / three fractions per week was useful for lung cancer $\geqslant 2 \mathrm{~cm}$ in size from a perspective of radiobiology [24]. Thus, the moderate hypo-fractionated SBRT of this study (56 Gy / seven fractions / three fractions per week) might also be useful for both elderly and central lung cancer patients. However, transient increase of tumor volume during SBRT might cause dose deficiency of edge parts of tumors. An accurate definition of targets and appropriate setting of PTV margins were required for effective SBRT. The SUVmax value might be useful for prediction of transient increase during SBRT.

\section{Conclusions}

In this study, variation of tumor volume during moderate hypo-fractionated SBRT for non-small cell lung cancer and metastatic lung cancer was investigated. As a result, a transient increase in tumor volume was found in an early stage of the treatment course. Especially if SUVmax value was $<9$, a transient increase during SBRT was likely to occur. Accurate prediction of tumor volume variation is required for more accurate treatment, such as adaptive radiation therapy.

\section{Additional Information \\ Disclosures}

Human subjects: Consent was obtained or waived by all participants in this study. Ethics Committee of the 
Saitama Medical Center, Saitama Medical University issued approval 1593. Animal subjects: All authors have confirmed that this study did not involve animal subjects or tissue. Conflicts of interest: In compliance with the ICMJE uniform disclosure form, all authors declare the following: Payment/services info: All authors have declared that no financial support was received from any organization for the submitted work. Financial relationships: All authors have declared that they have no financial relationships at present or within the previous three years with any organizations that might have an interest in the submitted work. Other relationships: All authors have declared that there are no other relationships or activities that could appear to have influenced the submitted work.

\section{References}

1. Timmerman R, Paulus R, Galvin J, et al.: Stereotactic body radiation therapy for inoperable early stage lung cancer. JAMA. 2010, 303:1070-6. 10.1001/jama.2010.261

2. 2) Nagata Y, Hiraoka M, Shibata T, et al.: Prospective Trial of Stereotactic Body Radiation Therapy for Both Operable and Inoperable T1N0M0 Non-Small Cell Lung Cancer: Japan Clinical Oncology Group Study JCOG0403. Int J Radiat Oncol Biol Phys. 20151, 93:989-96. 10.1016/j.ijrobp.2015.07.2278. Epub 2015 Nov 11

3. Hiraoka M, Ishikura S: A Japan clinical oncology group trial for stereotactic body radiation therapy of nonsmall cell lung cancer. J Thorac Oncol. 2007, 2:S115-7. 10.1097/JTO.0b013e318074de1b

4. Onishi H, Shirato H, Nagata Y, et al.: Hypofractionated stereotactic radiotherapy (HypoFXSRT) for stage I non-small cell lung cancer: updated results of 257 patients in a Japanese multi-institutional study. J Thorac Oncol. 2007, 2:S94-100. 10.1097/JTO.0b013e318074de34

5. Nagata Y, Takayama K, Matsuo Y, et al.: Clinical outcomes of a phase I/II study of 48 Gy of stereotactic body radiotherapy in 4 fractions for primary lung cancer using a stereotactic body frame. Int J Radiat Oncol Biol Phys. 2005, 63:1427-31. 10.1016/j.ijrobp.2005.05.034

6. 6) Onimaru R, Shirato H, Shimizu S, et al.: Tolerance of organs at risk in small-volume, hypofractionated, image-guided radiotherapy for primary and metastatic lung cancers. Int J Radiat Oncol Biol Phys. 20031, 56:126-35. 10.1016/s0360-3016(03)00095-6

7. Tatekawa K, Iwata H, Kawaguchi T, et al.: Changes in volume of stage I non-small-cell lung cancer during stereotactic body radiotherapy. Radiat Oncol. 2014, 9:8. 10.1186/1748-717X-9-8

8. 8) Gunter T, Ali I, Matthiesen C, et al.: Gross tumour volume variations in primary non-small-cell lung cancer during the course of treatment with stereotactic body radiation therapy. J Med Imaging Radiat Oncol. 2014, 58:384-91. 10.1111/1754-9485.12168. Epub 2014 Mar 5

9. Bhatt AD, El-Ghamry MN, Dunlap NE, et al.: Tumor volume change with stereotactic body radiotherapy (SBRT) for early-stage lung cancer: evaluating the potential for adaptive SBRT. Am J Clin Oncol. 2015, 38:41-6. 10.1097/COC.0b013e318287bd7f

10. 10) Lee YH, Kim YS, Lee HC, et al.: Tumour volume changes assessed with high-quality KVCT in lung cancer patients undergoing concurrent chemoradiotherapy. Br J Radiol. August. 2015, 88:20150156. 10.1259/bjr.20150156. Epub 2015 Jun 9

11. 11) Fox J, Ford E, Redmond K, et al.: Quantification of tumor volume changes during radiotherapy for nonsmall-cell lung cancer. Int J Radiat Oncol Biol Phys. 20091, 74:341-8. 10.1016/j.ijrobp.2008.07.063. Epub 2008 Nov 27

12. Tsakiridis T, Cutz JC, Singh G, et al.: Association of phosphorylated epidermal growth factor receptor with survival in patients with locally advanced non-small cell lung cancer treated with radiotherapy. J Thorac Oncol. 2008, 3:716-22. 10.1097/JTO.0b013e31817c6094

13. Gould MK, Maclean CC, Kuschner WG, Rydzak CE, Owens DK: Accuracy of positron emission tomography for diagnosis of pulmonary nodules and mass lesions: a meta-analysis. JAMA. 2001, 285:914-24. 10.1001/jama.285.7.914

14. 14) Shankar LK, Hoffman JM, Bacharach S, et al.: National Cancer Institute. Consensus recommendations for the use of 18F-FDG PET as an indicator of therapeutic response in patients in National Cancer Institute Trials. J Nucl Med. 2006, 47:1059-66.

15. Han B, Lin S, Yu LJ, Wang RZ, Wang YY: Correlation of ${ }^{18}$ F-FDG PET activity with expressions of survivin, Ki67, and CD34 in non-small-cell lung cancer. Nucl Med Commun. 2009, 30:831-7. 10.1097/MNM.0b013e32832dcfc4

16. 16) Lewis JH and Jiang SB: A theoretical model for respiratory motion artifacts in free-breathing CT scans . Phys Med Biol. 20097, 54:745-55. 10.1088/0031-9155/54/3/018. Epub 2009 Jan 9

17. 17) Lagerwaard FJ, Van Sornsen de Koste JR, Nijssen-Visser MR, et al.: Multiple "slow" CT scans for incorporating lung tumor mobility in radiotherapy planning. Int J Radiat Oncol Biol Phys. 200115, 51:932-7. 10.1016/s0360-3016(01)01716-3

18. 18) Sandhu AP, Lau SK, Rahn D, et al.: Stereotactic body radiation therapy in octogenarians with stage I lung cancer. Clin Lung Cancer. 2014, 15:131-5. 10.1016/j.cllc.2013.08.007. Epub 2013 Oct 21

19. 19) Mancini BR, Park HS, Harder EM, et al.: Elderly patients undergoing SBRT for inoperable early-stage NSCLC achieve similar outcomes to younger patients. Lung Cancer. 2016, 97:22-7.

10.1016/j.lungcan.2016.04.011. Epub 2016 Apr 19

20. 20) Haseltine JM, Rimner A, Gelblum DY, et al.: Fatal complications after stereotactic body radiation therapy for central lung tumors abutting the proximal bronchial tree. Pract Radiat Oncol. 2016, 6:27-33. 10.1016/j.prro.2015.09.012. Epub 2015 Nov 11

21. Tekatli H, Senan S, Dahele M, Slotman BJ, Verbakel WF: Stereotactic ablative radiotherapy (SABR) for central lung tumors: Plan quality and long-term clinical outcomes. Radiother Oncol. 2015, 117:64-70. 10.1016/j.radonc.2015.09.028

22. 22) Mangona VS, Aneese AM, Marina O, et al.: Toxicity after central versus peripheral lung stereotactic body radiation therapy: a propensity score matched-pair analysis. Int J Radiat Oncol Biol Phys. 2015, 91:124-132. 10.1016/j.ijrobp.2014.08.345. Epub 2014 Oct 18

23. 23) Kimura T, Nagata Y, Harada H, et al.: Phase I study of stereotactic body radiation therapy for centrally 


\section{Cureus}

located stage IA non-small cell lung cancer (JROSG 10-1). Int J Clin Oncol. 2017, 22:849-856.

10.1007/s10147-017-1125-y. Epub 2017 May 2

24. 24) Shibamoto Y, Miyakawa A, Otsuka S, et al.: Radiobiology of hypofractionated stereotactic radiotherapy: what are the optimal fractionation schedules?. J Radiat Res. 2016, 57:176-182. 10.1093/jrr/rrw015. Epub 2016 Mar 22 